\title{
Metalloproteinases, inflammation, and rheumatoid arthritis
}

\author{
F F Mohammed, D S Smookler, R Khokha
}

Ann Rheum Dis 2003;62(Suppl II):ii43-ii47

Ideally, the inflammatory response occurs rapidly to terminate infection. It also must halt in a timely manner to stop this reaction from inflicting self damage. Such a highly regulated process results from altering balances in proand anti-inflammatory signals orchestrated by multiple cell types and factors within the tissue microenvironment. The discovery of new substrates of metalloproteinases within this microenvironment has disclosed a new function in inflammation. The role of these proteases now extends beyond extracellular matrix remodelling enzymes to that of mediators of inflammatory signals involving various chemokines and cytokines. As natural inhibitors of these metalloproteinases, TIMPs have the potential of regulating the inflammatory response and affecting diseases such as rheumatoid arthritis. TIMP-3, in particular, stands out as an important regulator of inflammation with its ability to specifically inhibit proinflammatory cytokines and tissue destruction in the joint.

s nflammation is a coordinated response that protects and heals the host after infection or tissue damage and involves several molecular cues generated from either host or disease agent. ${ }^{1}$ Critical to the effectiveness of this reaction is the ability of the host response to halt once the insult has resolved, preventing self inflicted damage. This self regulation results from balances between pro- and anti-inflammatory signals. Often, some of the first respondents in an inflammatory reaction are resident mast cells and macrophages within the tissue. ${ }^{1}$ Upon stimulation, these cells release a variety of proinflammatory factors, including chemokines and cytokines, that trigger multiple activation cascades culminating in leucocyte infiltration, coagulation, tissue breakdown or liquefaction, and matrix destruction (fig 1). One can appreciate the need to control this reaction. Deregulated responses underlie many inflammatory diseases while the reverse condition, insufficient response, may lead to immunodeficiency.

Many of the inflammatory signals are orchestrated within the tissue microenvironment external to a cell. A superfamily of proteases called metzincins (zinc dependent metallopeptidases) includes the matrixin family of matrix metalloproteinases (MMPs) that are well known regulators of extracellular matrix (ECM) proteolysis in the microenvironment. Other members of the metzincin superfamily include the transmembrane proteases ADAMs (a disintegrin and metalloproteinases) and the secreted ADAMTSs (ADAM with thrombospondin domain). The discovery of MMP substrates beyond ECM proteins has disclosed a new role in inflammation. MMPs can cleave adhesion molecules, cytokines, chemokines, growth factors as well as their receptors, and binding proteins. ${ }^{23}$ Although not all ADAMs are proteolytically active, they are recognised for their ability to process or shed cell surface molecules, whereas ADAMTS enzymes can cleave matrix and other proteins. Tissue inhibitors of metalloproteinases
(TIMPs) are well known for their MMP inhibitory activity and there is now evidence that some TIMPs also inhibit specific ADAM and ADAMTS enzymes.

Many studies report the involvement of MMPs in inflammatory diseases such as those listed in table 1. Although the mechanisms are often not clear, MMP expression and activity usually correlates with severity in both autoimmune diseases and disorders with infectious origins. Furthermore, it has long been suggested that a link exists between chronic inflammation and cancer, ${ }^{4}$ and the importance of MMP activity in cancer has been well established. MMPs are traditionally thought of as tissue remodelling enzymes that are activated during an inflammatory response. Here we present a perspective on an emerging field that demonstrates how, in addition to their tissue destructive activity, metalloproteinases can modulate molecular mediators of inflammation, including generating both pro- and anti-inflammatory signals (fig 1).

\section{MMPS AS MEDIATORS OF INFLAMMATION BEYOND ECM DEGRADATION}

In contrast with MMP mediated tissue damage during inflammation, an example of a protective MMP response is the activation of the host bactericidal peptide $\alpha$-defensin by MMP-7 in the small intestine..$^{5}$ This antibacterial peptide can kill bacteria through membrane disruption and is a first defence in innate immunity. $\alpha$-Defensins are produced as propetides by mucosal epithelia, and proteolytic processing is required for their activation. MMP-7 deficient mice lack mature $\alpha$-defensin, leading to a greater number of surviving bacteria in the gut after oral administration. Interestingly, MMP-7 deficient mice showed no defects in connective tissue remodelling, suggesting that MMPs can function independently of their ECM degrading activity.

The infiltration of inflammatory cells after injury is directed by signals generated from epithelial cells in the form of chemokine gradients. Chemokines are divided into four groups depending on the positions of their cysteine residues (CXC, CC, C, and CXXXC) and attract inflammatory cells by binding to chemokine receptors. Binding causes rapid alterations in cell adhesion molecules that facilitate inflammatory cell adhesion to endothelial cells. These cells migrate along a gradient of increasing chemokine concentration to the site of origin. One such CXC chemokine called KC (CXCL1) is thought to bind the cell surface proteoglycan, syndecan-l. MMP-7 is secreted by injured epithelial cells and cleaves the

Abbreviations: ADAM, a disintegrin and metalloproteinase; ADAMTS, ADAM with thrombospondin domain; ECM, extracellular matrix; IL, interleukin; $M C P$, monocyte chemoattractant protein; MMPs, matrix metalloproteinases; RA, rheumatoid arthritis; SDF, stromal cell derived factor, TACE, TNF $\alpha$ converting enzyme; TGF $\beta$, transforming growth factor $\beta$; TIMPs, tissue inhibitors of matrix metalloproteinases; TNF $\alpha$, tumour necrosis factor $\alpha$ 


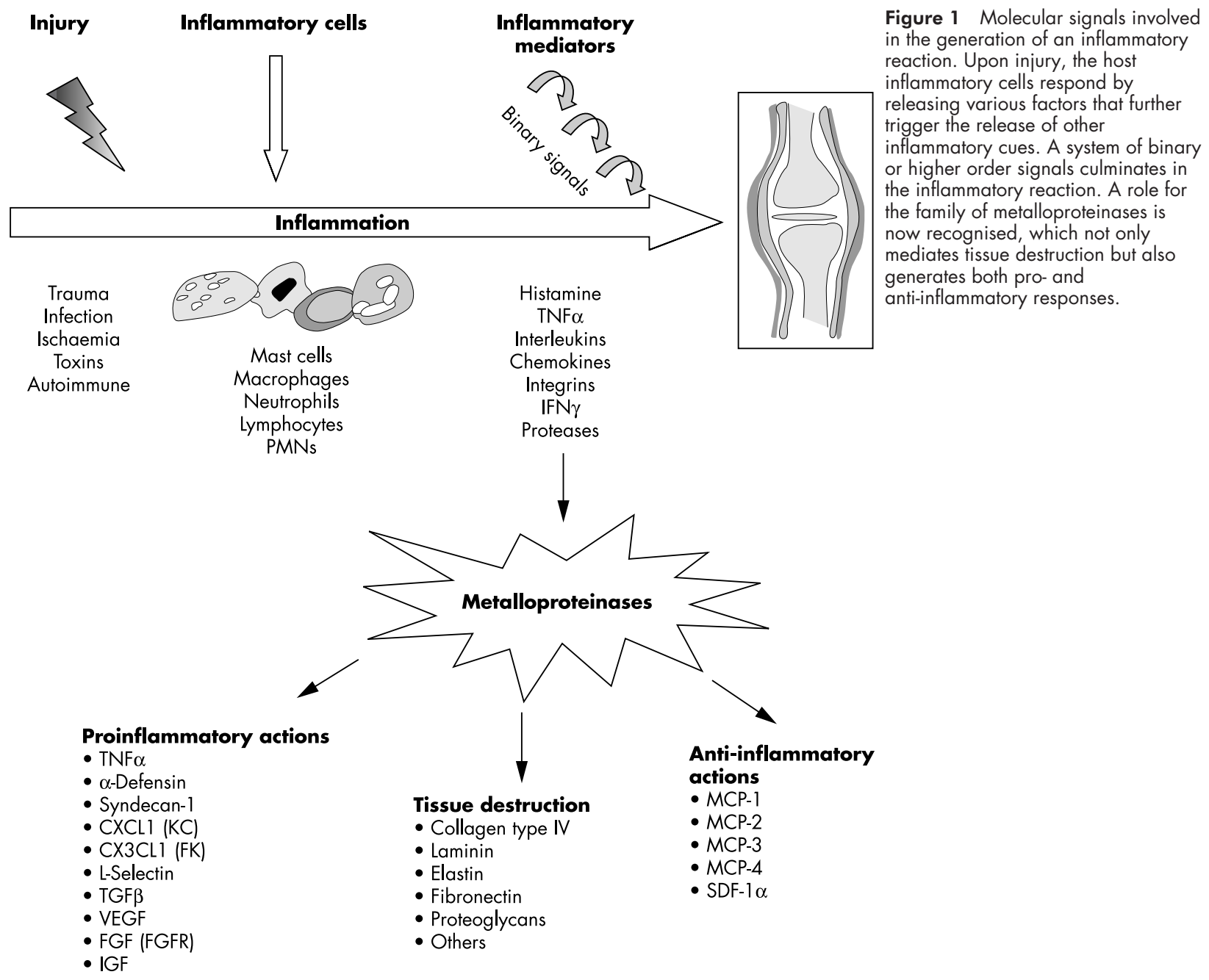

ectodomain of syndecan-1 in an in vivo lung injury model. ${ }^{6}$ The shedding of syndecan-1 releases $\mathrm{KC}$, which then stimulates trans-epithelial migration of inflammatory cells into the alveolar space. These MMP-7 deficient mice, which are protected against severe lung injury due to impaired neutrophil infiltration, illustrate that MMP mediated processing can have a stimulatory role during inflammation.

MMPs may in fact have a dual effect on chemokines, as proteolytic cleavage can inactivate some chemokines while activating others. MMP-1, MMP-2, MMP-13, and MMP-14, but not MMP-7, cleave monocyte chemoattractant protein-3 (MCP-3), thereby inactivating this CC chemokine that is

Table 1 Inflammatory disorders in which MMPs are implicated

\begin{tabular}{ll}
\hline Alzheimer's disease & Psoriasis \\
Asthma & Rheumatoid arthritis \\
Atherosclerosis & Sarcoidosis \\
Atopic dermatitis & Systemic lupus erythematosus \\
Bullous pemphigoid & Type I diabetes mellitus \\
Chronic obstructive pulmonary disease & Ulcerative colitis \\
Gout & Helicobacter pylori gastritis* \\
Inflammatory bowl disease & Hepatitis C* \\
Ischaemia-reperfusion injury & Neisserial or pneumococcal \\
Multiple sclerosis & meningitis* \\
Osteoarthritis & Tuberculosis* \\
\hline
\end{tabular}

*Diseases of infectious origin in which host inflammatory reaction is as important to its pathology as the microbial infection itself. Adapted from Nathan. known to recruit monocytes and leucocytes. ${ }^{78}$ Similarly, MCP-1, MCP-2, and MCP-4 are inactivated through cleavage by MMP-1 and MMP-3. ${ }^{8}$ This type of regulation is also seen with the CXC chemokine, stromal cell derived factor (SDF)- $1 \alpha$, which can be cleaved by MMP-1, MMP-2, MMP-3, MMP-9, MMP-13, and MMP-14. ${ }^{9}$ The cleaved SDF- $1 \alpha$ can no longer bind to its chemokine receptor. Interestingly in the above studies, MMP-7 does not cleave and therefore cannot inactivate MCPs or SDF- $1 \alpha$. Thus, specific MMPs may serve as proinflammatory mediators, whereas others serve to dampen inflammation. Collectively, these studies highlight the requirement for tight regulation of the metalloproteinase proteolytic axis to ensure appropriate start and stop signals during the inflammatory reaction.

\section{TIMP-3 AND TACE AS INFLAMMATORY REGULATORS}

The proteolytic axis in any biological system relies on a delicate balance between proteinases and their endogenous inhibitors. Thus it is also important to understand how the inhibitors function in opposing the proteolytic events that occur during the inflammatory response. To date, there are four identified TIMP proteins (TIMP-1 to TIMP-4) that share many similarities. ${ }^{10}$ They are expressed in a wide variety of tissues and biological systems. Of the four TIMPs, TIMP-3 has a number of unique features. In contrast with the other soluble TIMPs, TIMP-3 is secreted and binds to the ECM. Binding can occur with the ECM proteins such as heparan sulphate and 


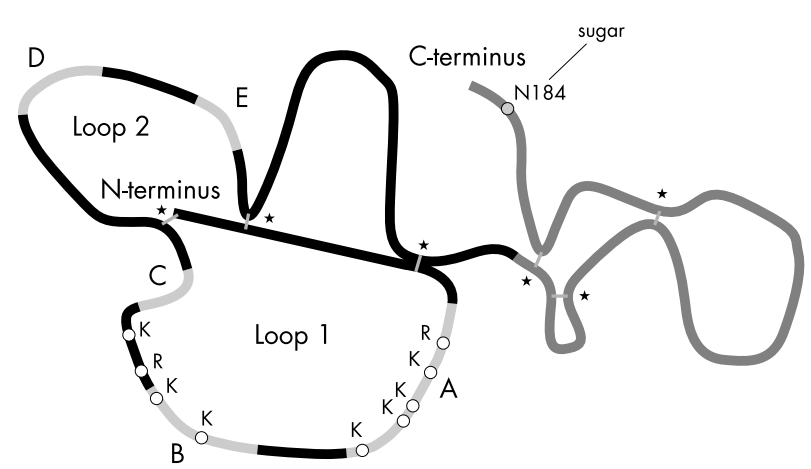

Figure 2 Structure of the TIMP-3 protein. TIMP-3 is glycosylated at the asparagine amino acid, N184 and contains six disulphide bonds marked by asterisks. The gray sections represent the five strands ( $A-E)$ of the $N$-terminus $\beta$-barrel. Strands $A$ and $B$ can each strongly bind heparan sulphate, which is suggested to result from the net positive charge in these regions. They contain nine basic amino acids indicated by open circles and only one acidic residue. TIMP-1 and TIMP-2 contain several acidic residues that neutralise the charge from their basic residues residing in this region.

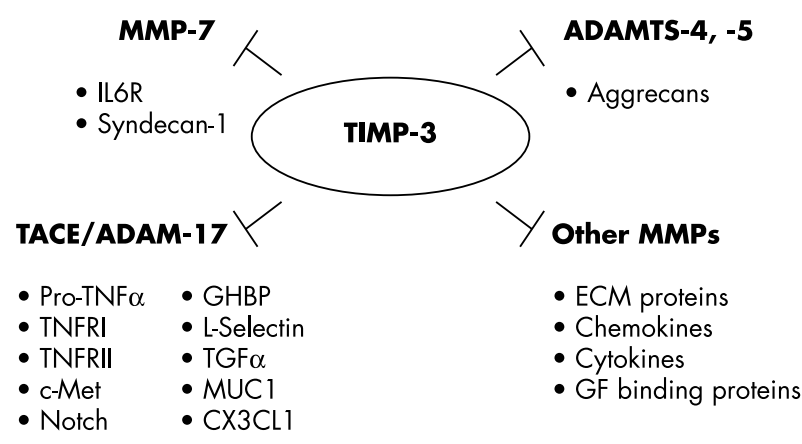

Figure 3 The broader inhibition profile of TIMP-3. TIMP-3 stands unique among the TIMP family of inhibitors, as it can inhibit ADAM and ADAMTS enzymes in addition to MMPs. These metalloproteinases are known to proteolytically process several factors involved in the normal inflammatory response and in inflammatory diseases.

chondroitin sulphate, through the N-terminal heparin binding domains of TIMP-3 rich in lysine and arginine ${ }^{11}$ (fig 2). These domains are considerably more basic than those in the more readily soluble TIMP- 1 and TIMP-2. The excess positive charge in the TIMP-3 domains may interact with sulphate groups present on the heparin backbone. However, there is evidence to suggest that the C-terminal of TIMP-3 can also bind the ECM, although the specific domains involved are not known. ${ }^{12}$ The importance of TIMP-3 is highlighted by the fact that TIMP-3 is the only TIMP genetically linked to a human disorder, Sorsby's fundus dystrophy, a degenerative disease of the retina. ${ }^{13}$ The unique feature of TIMP-3 most relevant to inflammation is its broader inhibition profile. Figure 3 summarises the substrates of these TIMP-3 targeted proteases. Several of these substrates are inherently linked to the inflammatory reaction.

In addition to MMPs, TIMP-3 can inhibit ADAM-17 (also called tumour necrosis factor $\alpha$ (TNF $\alpha)$ converting enzyme, TACE) ${ }^{14}$ and ADAMTS- 4 and -5 (aggrecanases). ${ }^{15}$ As the only known biochemical inhibitor of the sheddase TACE, ${ }^{14}$ TIMP-3 may be a key regulator of TNF $\alpha$ levels. TACE is responsible for processing membrane bound TNF $\alpha$ to its soluble form. ${ }^{16}{ }^{17}$ The shed TNF $\alpha$ becomes locally and systemically available and is a major proinflammatory cytokine produced by activated macrophages. TNF $\alpha$ signals mainly through its type I receptor, TNFRI, and initiates a cascade of other cytokines (such as interleukin (IL)6) ${ }^{18}$ and chemokines (such as MCP-1), ${ }^{19}$ as well as inducing MMP transcription..$^{20}$ Notably, TACE also sheds the TNF $\alpha$ receptors, TNFRI and TNFRII. ${ }^{21}{ }^{22}$ Soluble TNFRII is known to act as a decoy by binding soluble TNF $\alpha$, which prevents binding and signalling of TNFRI. ${ }^{23}$ Thus TIMP-3, unlike the other TIMPs, is positioned to have an impact on the TNF $\alpha$ signalling system. Altogether, TIMP-3 may influence molecular cues that initiate the inflammatory reaction as well as the remodelling process through inhibition of MMP and aggrecanase activity.

\section{THE METALLOPROTEINASE AXIS IN RHEUMATOID ARTHRITIS (RA)}

The articular cartilage of the joint is made up of collagen type II, which provides tensile strength, as well as proteoglycan aggregates that create bulk. These aggregates consist of multiple units of the core protein called aggrecan, attached to many molecules of chondroitin sulphate or keratin sulphate. About 100 of these proteoglycans are covalently linked to the sulphated glycosaminoglycans to form a single aggregate. The integrity of the joint structure is dependent on these prevalent, high molecular weight aggregates. Aggrecanase-1 and -2 (ADAMTS-4 and ADAMTS-5) are the principal proteases involved in aggrecan degradation. Aggrecanases bind to the sulphated glycosaminoglycans through their type-I thrombospondin motifs, ${ }^{25}$ helping them localise to the proteoglycan to cleave the core protein, ${ }^{26}$ and have been found to be increased in both RA and osteoarthritis. ${ }^{27}$ TIMP-3 is about 50 times more effective at inhibiting aggrecanase- 1 than TIMP- 1 or TIMP- $2 .{ }^{28}$ Aside from the aggrecanases, MMPs participate in joint deterioration through the cleavage of aggrecan. For instance, MMP-3 can cleave aggrecan between amino acids N341 and F342, an activity strongly inhibited by TIMP-3. ${ }^{29}$ Therefore TIMP-3, which is normally expressed in the cartilage, can directly inhibit both aggrecanases and MMPs, preventing aggrecan degradation.

The other major component of the joint cartilage, collagen, is also subject to proteolytic degradation by MMPs. The collagenase MMP-13 preferentially targets the triple helix of collagen type II, and the released monomer fragments are subsequently degraded by the gelatinases MMP-2 and MMP$9{ }^{25}$ Among the TIMPs, TIMP-3 is the most effective inhibitor of MMP- $13^{30}$ and can also inhibit MMP-2 and MMP-9. Thus TIMP-3 appears to be ideally situated to exert a broad impact on controlling joint degradation.

Upstream of these events are the cytokine signals that trigger inflammation. An imbalance of proinflammatory and anti-inflammatory cytokines contributes to the pathology of arthritis. ${ }^{31}$ Many different cytokines have been described in RA and among these, TNF $\alpha$ and ILl seem to be the major proinflammatory cytokines ${ }^{31}$ and are produced by macrophages in the inflamed synovium. As discussed above, TNF $\alpha$ levels may be regulated by TACE, suggesting a role for TIMP-3 in inhibition of TNF $\alpha$ induced cascades of downstream inflammatory cytokines in RA. Thus, the cytokine imbalance seen in RA may result in part from altered balances in the MMP/ADAM/ ADAMTS/TIMP proteolytic axis (fig 4). Furthermore, TNF $\alpha$ itself can induce the synthesis and secretion of MMPs, which in turn affect chemokine and cytokine action. Conceivably, a positive feedback loop exists between cytokines and metalloproteinase activities, creating a vicious cycle of destruction.

TIMP-3 may additionally affect the influx of inflammatory cells through TACE activity during inflammation. For example, fractalkine (FK, CXXXCLl) is a chemokine that is normally bound to the cell membrane and functions as a cell adhesion molecule for circulating inflammatory cells. TACE is known to mediate FK shedding from the cell surface, allowing it to act as a potent chemoattractant. ${ }^{32}$ Soluble levels of FK are also raised in the synovial fluid of patients with RA. ${ }^{33}$ Another chemotactic factor for monocytes in RA is transforming 


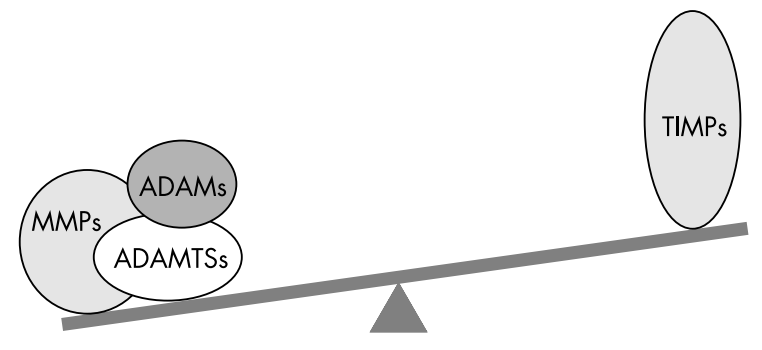

Proteolytic axis

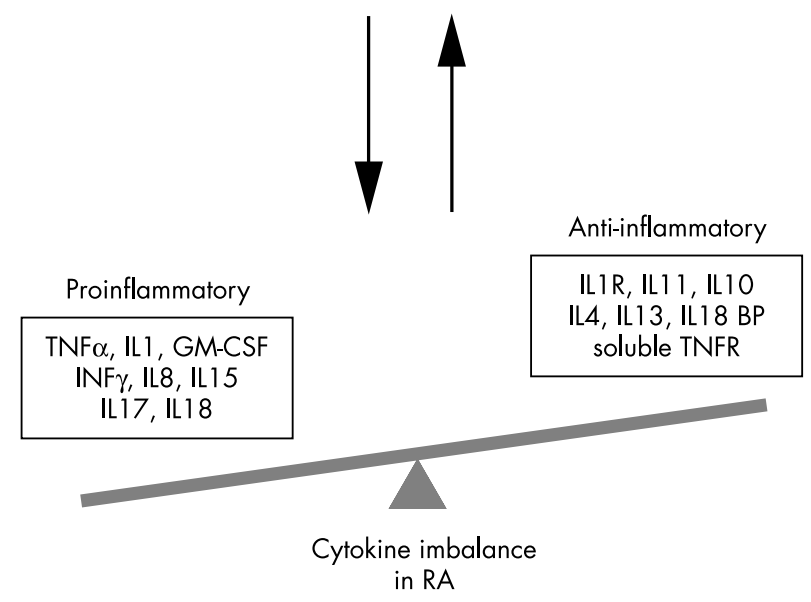

Figure 4 The projected interactions between the metalloproteinase axis and the cytokine axis in rheumatoid disease. Metalloproteinase activity is linked to the activation of various cytokines involved in inflammation. The net metalloproteinase activity can shift the balance between some pro- and anti-inflammatory cytokines. Cytokines, in turn, also induce the production and activation of various metalloproteinases. An imbalance in one axis can potentially cause imbalance in the other perpetuating a loop of destructive activity in RA.

growth factor $\beta$ (TGF $\beta$ ). MMP-2, MMP-3, MMP-7, and MMP-9 have been shown to release TGF $\beta$ from the matrix, allowing for its activation. ${ }^{3}$ Finally, angiogenesis also occurs at the site of inflammation and it has been shown that MMPs regulate the bioavailability of several angiogenic factors such as vascular endothelial growth factor (VEGF), fibroblast growth factor (FGF) receptor, epithelial growth factor (EGF), insulin-like growth factor (IGF), and TGF $\beta .^{23}$ Recently, TIMP- 1 and TIMP- 3 overexpression by adenoviral gene transfer was shown to reduce the invasion and proliferation of rheumatoid synovial fibroblasts. ${ }^{34}$ An inhibitory function of TIMPs is thus possible at several levels and pathways during the development and progression of RA.

\section{SUMMARY}

Metalloproteinases have a fundamental role during the inflammatory response. This is becoming evident by their influence on the molecular cues involved in the initiation and dampening of inflammation. Proteolytic activity can regulate the pro- and anti-inflammatory cascades, as well as the process of tissue remodelling. Cleavage of chemokines, cytokines, and their receptors can activate or inactivate these critical signals and thus may exert dual effects. Furthermore, it appears that the cytokine and metalloproteinase axes are intrinsically linked where the balance of one can influence the other. This, together with the ability of MMPs and aggrecanases to degrade matrix/cartilage, highlights the complex role of these pericellular enzymes in the inflammatory reaction. Of the inhibitors, TIMP-3 is intriguing in its ability to block the activity of MMP, ADAM, and ADAMTS enzymes. Further research will disclose the physiological importance of the MMP/ADAM/ADAMTS/TIMP proteolytic axis during the inflammatory response.

\section{Authors' affiliations \\ F F Mohammed, D S Smookler, R Khokha, Ontario Cancer}

Institute/University Health Network, Toronto, Canada

Correspondence to: Professor R Khokha, Ontario Cancer

Institute/University Health Network, 610 University Avenue, Toronto,

M5G 2 M9 Canada; rkhokha@uhnres.utoronto.ca.

\section{REFERENCES}

1 Nathan C. Points of control in inflammation. Nature 2002;420:846-52.

2 Vu TH, Werb Z. Matrix metalloproteinases: effectors of development and normal physiology. Genes Dev 2000; 14:2123-33.

3 Hojilla C, Mohammed F, Khokha R. Directing cell fate through matrix metalloproteinases during cancer progression. Br J Cancer (in press).

4 Coussens LM, Werb Z. Inflammation and cancer. Nature 2002;420:860-7

5 Wilson CL, Ouellette AJ, Satchell DP, Ayabe T, Lopez-Boado YS, Stratman JL, et al. Regulation of intestinal alpha-defensin activation by the metalloproteinase matrilysin in innate host defense. Science 1999;286:113-17.

6 Li Q, Park PW, Wilson CL, Parks WC. Matrilysin shedding of syndecan-1 regulates chemokine mobilization and transepithelial efflux of neutrophils in acute lung injury. Cell 2002;111:635-46.

7 McQuibban GA, Gong JH, Tam EM, McCulloch CA, Clark-Lewis I, Overall CM. Inflammation dampened by gelatinase A cleavage of monocyte chemoattractant protein-3. Science 2000;289:1202-6.

8 McQuibban GA, Gong JH, Wong JP, Wallace JL, Clark-Lewis I, Overall CM. Matrix metalloproteinase processing of monocyte chemoattractant proteins generates $\mathrm{CC}$ chemokine receptor antagonists with anti-inflammatory properties in vivo. Blood 2002;100:1 160-7.

9 McQuibban GA, Butler GS, Gong JH, Bendall L, Power C, Clark-Lewis I, et al. Matrix metalloproteinase activity inactivates the CXC chemokine stromal cell-derived factor-1. J Biol Chem 2001;276:43503-8.

10 Brew K, Dinakarpandian D, Nagase H. Tissue inhibitors of metalloproteinases: evolution, structure and function. Biochim Biophys Acta 2000; 1477:267-83.

11 Yu WH, Yu S, Meng Q, Brew K, Woessner JF Jr. TIMP-3 binds to sulfated glycosaminoglycans of the extracellular matrix. J Biol Chem 2000;275:31226-32.

12 Langton KP, Barker MD, McKie N. Localization of the functional domains of human tissue inhibitor of metalloproteinases- 3 and the effects of Sorsby's fundus dystrophy mutation. J Biol Chem 1998;273:1677881

13 Weber BHF, Vogt G, Pruett RC, Stohr H, Felbor U. Mutations in the tissue inhibitor of metalloproteinases-3 (TIMP3) in patients with Sorsby's fundus dystrophy. Nat Genet 1994:8:352-6.

14 Amour A, Slocombe PM, Webster A, Butler M, Knight CG, Smith BJ, et al. TNF-alpha converting enzyme (TACE) is inhibited by TIMP-3. FEBS Lett 1998:435:39-44.

15 Kashiwagi $M$, Tortorella M, Nagase H, Brew K. TIMP-3 is a potent inhibitor of aggrecanase 1 (ADAM-TS4) and aggrecanase 2 (ADAM-TS5). Biol Chem 2001;276:12501-4.

16 Black RA, Rauch CT, Kozlosky CJ, Peschon JJ, Slack JL, Wolfson MF, et al. A metalloproteinase disintegrin that releases tumour-necrosis factor alpha from cells. Nature 1997:385:729-33.

17 Moss ML, Jin SL, Milla ME, Bickett DM, Burkhart W, Carter HL, et al. Cloning of a disintegrin metalloproteinase that processes precursor tumour-necrosis factor-alpha. Nature 1997;385:733-6.

18 Butler D, Maini RN, Feldmann M, Brennan FM. Blockade of TNF $\alpha$ with chimeric anti-TNF $\alpha$ monoclonal antibody, cA2 reduces (IL-6 and IL-8) release in RA NMC cultures: a comparison with IL-1 ra. Eur Cytokin Netwk 1995;6:225-30.

19 Bian ZM, Elner SG, Strieter RM, Kunkel SL, Lukacs NW, Elner VM. IL-4 potentiates IL-beta-and TNF-alpha-stimulated IL-8 and MCP-I protein production in human retinal pigment epithelial cells. Curr Eye Res 1999; 1 8:349-57.

20 Borden P, Heller RA. Transcriptional control of matrix metalloproteinases and the tissue inhibitors of matrix metalloproteinases. Crit Rev Eukaryot Gene Exp 1997;7:159-78.

21 Peschon JJ, Slack JL, Reddy P, Stocking KL, Sunnarborg SW, Lee DC, et al. An essential role for ectodomain shedding in mammalian development, Science 1998282:1281-4.

22 Reddy P, Slack JL, Davis R, Cerretti DP, Kozlosky CJ, Blanton RA, et al. Functional analysis of the domain structure of tumor necrosis factor-alpha converting enzyme. J Biol Chem 2000;275:14608-14.

23 Aderka D, Engelmann H, Maor Y, Brakebusch C, Wallach D. Stabilization of the bioactivity of tumor necrosis factor by its soluble receptors. J Exp Med 1992;175:323-9.

24 Higuchi M, Aggarwal BB. Modulation of two forms of tumor necrosis factor receptors and their cellular response by soluble receptors and their monoclonal antibodies. J Biol Chem 1992;267:20892-9. 
25 Mort JS, Billington CJ. Articular cartilage and changes in arthritis: matrix degradation. Arthritis Res 2001;3:337-41.

26 Arner EC, Hughes CE, Decicco CP, Caterson B, Tortorella MD. Cytokine-induced cartilage proteoglycan degradation is mediated by aggrecanase Osteoarthritis Cartilage 1998;6:214-28.

27 Murphy G, Knauper V, Atkinson S, Butler G, English W, Hutton M, et al. Matrix metalloproteinases in arthritic disease. Arthritis Res 2002;4(suppl):S39-49.

28 Hashimoto G, Aoki T, Nakamura H, Tanzawa K, Okada Y. Inhibition of ADAMTS4 (aggrecanase-1) by tissue inhibitors of metalloproteinases (TIMP-1, 2, 3 and 4). FEBS Lett 2001;494:192-5.

29 Apte SS, Olsen BR, Murphy G. The gene structure of tissue inhibitor of metalloproteinases (TIMP)-3 and its inhibitory activities define the distinct TIMP gene family. J Biol Chem 1995;270:14313-18.
30 Knauper V Lopez-Otin C, Smith B, Knight G, Murphy G. Biochemical characterization of human collagenase-3. J Biol Chem 1996;271:1544-50.

31 Arend WP. Physiology of cytokine pathways in rheumatoid arthritis. Arthritis Rheum 2001;45:101-6.

32 Garton KJ, Gough PJ, Blobel CP, Murphy G, Greaves DR, Dempsey PJ, et al. Tumor necrosis factor-alpha-converting enzyme (ADAM17) mediates the cleavage and shedding of fractalkine (CX3CL1). J Biol mediates the cleavage and sheddin

33 Ruth JH, Volin MV, Haines III GK, Woodruff DC, Katschke Jr KJ, Woods $J M$, et al. Fractalkine, a novel chemokine in rheumatoid arthritis and in rat adjuvant induced arthritis. Arthritis Rheum 2001;44:1568-81.

34 van der Laan WH, Quax PH, Seemayer CA, Huisman LG, Pieterman EJ, Grimbergen JM, et al. Cartilage degradation and invasion by rheumatoid synovial fibroblasts is inhibited by gene transfer of TIMP-1 and TIMP-3. Gene Ther 2003; 10:234-42.

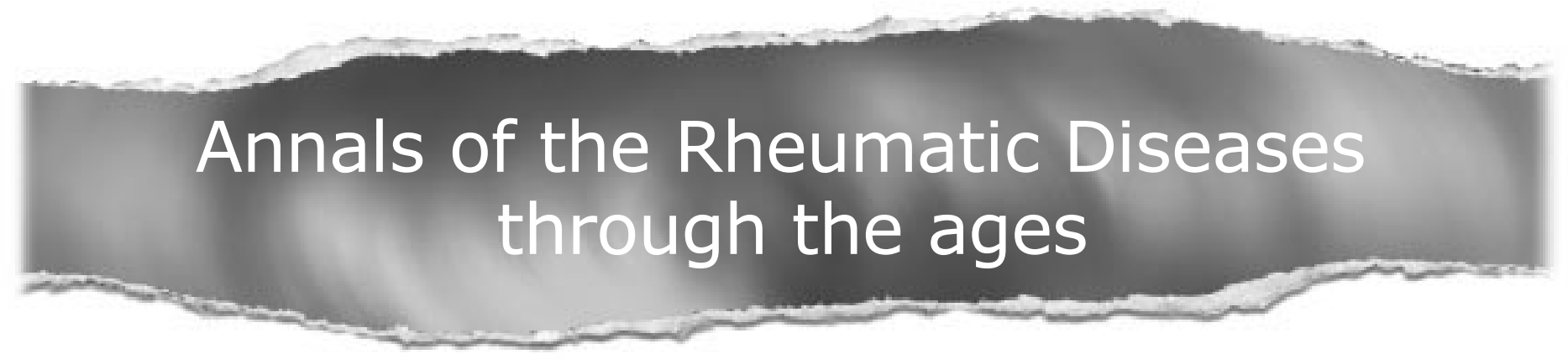

\section{Browse the Archive}

Annals of the Rheumatic Diseases online has an archive of content dating back to 1965 . Full text from 1997; abstracts from 1975; table of contents from 1965

\section{www.annrheumdis.com}

